\title{
RANGING SIGNAL DESIGN AND ITS DETECTION FOR OFDMA SYSTEMS
}

\author{
Kuhn Chang Lin and Yu T. Su \\ Department of Communications Engineering \\ National Chiao Tung University, Hsinchu, 30056 TAIWAN \\ Email addresses: tony.cm95g@nctu.edu.tw, ytsu@mail.nctu.edu.tw
}

\begin{abstract}
A single-symbol based initial ranging signal structure and the associated signal detection and timing synchronization methods for OFDMA systems are presented. The proposed structure makes it feasible and flexible for a receiver to detect single and multiple ranging codes and estimate the corresponding timing offsets. Our approach offers improved performance and enhanced robustness against multi-user interference and multi-path fading. Numerical results verify the effectiveness of the proposed method and its advantages over existing alternatives.
\end{abstract}

Index Terms - OFDMA, ranging process, multi-user ranging code detection, timing estimation, and MUSIC

\section{INTRODUCTION}

Several studies on the initial ranging for an OFDMA system like that specified by the IEEE 802.16e standard [1] have been reported [2]-[6]. These works fall into two major categories. The first category [2] is based on the ranging codes given in [1]. The second category [3]-[6] proposes new ranging code structures. [4] suggested an interleaved channel assignment scheme to overcome the major drawbacks of the above approaches. However, multiple OFDMA symbols are needed and the carrier frequency offsets (CFOs) between the received signals and the BS local reference is not taken into account and perfect frequency alignment among all ranging subscriber stations (RSSs) and the BS is assumed instead. Such an assumption is not realistic and ignores the fact that the residual CFOs cause the loss of orthogonality among ranging codes and are likely to induce severe performance degradation. Lin [5] and Sanguinetti [6] employed a MUSIC-like algorithm to overcome this difficulty. The latter, however, requires a longer ranging signal duration (and therefore lower system throughput). Guard bands between neighboring subbands of different ranging channels are also needed to prevent interference from other groups. We propose a specific ranging channel assignment and a new class of frequency domain ranging codes which enables the BS

This work is an extension of the IEEE $802.16 \mathrm{~m}$ technical contribution [5] and was supported in part by the NCTU-MediaTek Research Center at the National Chiao Tung University. to employ a MUSIC-like algorithm to detect multiple RSSs and estimate their timing offsets. Our design uses only one OFDM symbol; guard bands between ranging channels are not needed and the associated receive algorithm gives robust, excellent multi-user detection and timing estimation performance. Compared with the existing solutions, our proposal is more spectral efficient, yields improved performance and offers greater immunity against the multiuser interference and frequency selective fading.

Notation: [ ] and $j$ denote the floor operation and $\sqrt{-1}$. $(.)^{\mathbf{T}}$ and $(.)^{\mathbf{H}}$ denote transpose and Hermitian operations.

\section{CHANNEL ASSIGNMENT SCHEME}

Subcarriers assigned for RSSs are divided into $N_{g p}$ groups with each group having $N_{b}$ subbands and each subband consisting of $N_{c}$ consecutive subcarriers such that each group can support a maximum of $M$ RSSs. Let $S_{p}$ be the set of indices of the subcarriers allocated to the $p$ th group and denote by $R_{i p}$ the $i$ th RSS in the $p$ th group which employs the frequency domain ranging code $\left\{C_{i p}(u): u=1, \cdots, N_{b} N_{c}\right\}$. Let $f_{s}$ be the lowest subcarrier index allocated for RSSs. For $0 \leq p \leq N_{g p}-1, S_{p}$ is $S_{p}=\left\{f_{s}+p N_{c}+\iota D_{b}+\nu: 0 \leq \iota \leq N_{b}-1,0 \leq \nu \leq N_{c}-1\right\}$, where $D_{b}$ is the frequency spacing between two neighboring subbands. The frequency domain ranging signal $X_{i p}(k)$ for $R_{i p}$ is defined as

$$
X_{i p}(k)=\left\{\begin{array}{cc}
A_{i p} C_{i p}(u), & k \in S_{p}, u=1,2, \cdots N_{b} N_{c} \\
0, & \text { otherwise },
\end{array}\right.
$$

where $\left|C_{i p}(u)\right|=1$ and $A_{i p}$ is the relative amplitude of $R_{i p}^{(2)}$.

The maximum transmission delay $D_{\max }$ (samples) equals to the round-trip propagation delay between the BS and a RSS at the boundary of the cell. To avoid inter subcarrier interference (ICI) and inter symbol interference (ISI), the length of the cyclic prefix $(\mathrm{CP}), N_{g}$, must be larger than the sum of $D_{\max }$ and the maximum delay spread among all uplink ranging channels $L$ (samples) [7]. This assumption is not restrictive, since in many standardized OFDM systems the initialization blocks are usually preceded by long CPs. With the $\mathrm{CP}$ inserted, the time domain ranging signal for $R_{i p}$ is $x_{i p}(n), n=-N_{g},-N_{g}+1, \ldots, N$, where $N$ is the FFT size. 
Assuming the uplink channels remain static within a symbol duration and ignoring the presence of noise for the moment, we express the received ranging waveform $y_{i p}^{R}(n)$ for $R_{i p}$ as

$$
y_{i p}^{R}(n)=\sum_{l=0}^{L-1} h_{i p}(l) x_{i p}(n-l)
$$

where $h_{i p}(l), l=0, \cdots, L-1$ are the associated channel tap weights. The remaining $N-N_{g p} N_{c} N_{b}$ subcarriers are assigned to $N_{D S S}$ data subscriber stations (DSSs) which have already completed their initial ranging process and are assumed to be perfectly synchronized with the BS' time and frequency scales. $y_{i}^{D}(n)$ denotes the signal of the $i$ th DSS. The received signal at the $\mathrm{BS}$ thus becomes

$$
\begin{aligned}
y(n)= & \sum_{p=0}^{N_{g p}-1} \sum_{i=0}^{M-1} y_{i p}^{R}\left(n-d_{i p}\right) e^{j 2 \pi \varepsilon_{i p} n / N} \\
& +\sum_{i=0}^{N_{D S S S}-1} y_{i}^{D}(n)+w(n)
\end{aligned}
$$

where $\{w(n)\}$ are independent and identical distributed (i.i.d.) complex circular symmetric Gaussian random variables with mean zero and variance $\sigma_{w}^{2}=E\left[|w(n)|^{2}\right]$ and $d_{i p}, \varepsilon_{i p}$ represent the timing offset and normalized frequency offset of $R_{i p}$.

\section{RANGING SIGNAL STRUCTURE}

The maximum number of RSSs supported by one group, $M$, is $M=\min \left\{\left[N / N_{g}\right], \min \left\{N_{b}, N_{c}\right\}-1\right\}$. In practice, $\left[N / N_{g}\right]$ is usually larger than 2 , hence a judicial choice of $\left\{N_{b}, N_{c}\right\}$ is needed to ensure $M>2$.

The ranging code in the $\iota$ th subband, $C_{i p}^{\iota}$, for the $R_{i p}$ is given by

$$
C_{i p}^{L}(\nu)=e^{-j 2 \pi i \nu / M}, \nu=0,1, \cdots, N_{c}-1 .
$$

The same ranging code can be transmitted using different subcarrier group without causing any interference for the subcarriers allocated are disjoint. Our design does not ensure orthogonality among codes in the same group. When choosing the $i$ th ranging code, the phase difference between two consecutive assigned subcarriers are rotated by $-2 \pi i / M$. The phase rotation caused by round-trip delay and channel delay spread is between 0 and $-2 \pi / M$. This implies that the overall frequency-domain phase shifts of all RSSs in the same group are non-overlapping over $\left[\frac{-2 i \pi}{M}, \frac{-2(i+1) \pi}{M}\right]$, where $i=0,1, \cdots, M-1$. By using the MUSIC algorithm [?], we perform one-to-one mapping from the overall phase shifts of RSSs in the same group into the corresponding RSSs associated with the detected peak positions. We can simultaneously obtain the delay information of $R_{i p}$ from the overall phase shift without additional complicated computations. The RSSs in the same group can thus be easily detected and decoupled without using multiple OFDMA symbols.

\section{RANGING METHOD}

Our estimate assume that the timing offset $d_{i p}$ for $R_{i p}$ is equal to the sum of the round-trip transmission delay and the channel group delay. When a RSS receives the timing offset estimate from the BS, it adjusts its timing accordingly and retransmit its ranging code with both cyclic prefix and postfix to avoid inter carrier interference (ICI).

The code structure of (5) and the assumption that the subband bandwidth is smaller than the uplink channel's coherent bandwidth implies that the equivalent channel gains within a the $\iota$ th subband of $R_{i p}$ are related by

$$
H_{i p}^{\iota}(\nu) \approx H_{i p}^{\iota}(0) e^{-j 2 \pi \nu\left(\frac{i}{M}+\frac{d_{i p}}{N}\right)}, \nu=1, \cdots, N_{c}-1
$$

Let $\mathbf{Y}_{p}^{\iota}$ be the $N_{c}$-dimensional vector that collects the components of the DFT output vector corresponding to the $\iota$ th subband of the $p$ th group. Since the DSSs are assumed to be perfectly synchronized to the BS time reference, their signals will not contribute to $\mathbf{Y}_{p}^{\iota}$, we have

$$
\mathbf{Y}_{p}^{\iota}=\sum_{i=0}^{M-1} \mathbf{F}\left(\varepsilon_{i p}\right) \mathbf{v}_{i p} H_{i p}^{\iota}(0)+\mathbf{n}_{p}^{\iota}
$$

where $\mathbf{n}_{p}^{\iota}$ is the sum of white Gaussian noise and the interference from the nearby groups and $\mathbf{v}_{i p}$ is given by

$\mathbf{v}_{i p}=\left(1, e^{-j 2 \pi\left(\frac{i}{M}+\frac{d_{i p}}{N}\right)}, \cdots, e^{-j 2 \pi\left(N_{c}-1\right)\left(\frac{i}{M}+\frac{d_{i p}}{N}\right)}\right)^{\mathbf{T}}$.

Furthermore, $\mathbf{F}\left(\varepsilon_{i p}\right)$ is a $N_{c} \times N_{c}$ Toeplitz matrix with the element in $a$ th row and the $b$ th column given by [7]

$$
f_{a, b}\left(\varepsilon_{i p}\right)=\frac{\sin \left(\pi\left(a-b+\varepsilon_{i p}\right)\right)}{N \sin \left(\pi\left(b-a+\varepsilon_{i p}\right) / N\right)} e^{j \pi\left(b-a+\varepsilon_{i p}\right)(N-1) / N}
$$

A RSS intending to start initial ranging should first derive its initial frequency and timing estimates from a downlink control signal broadcasted by the BS. This means the CFOs are only due to Doppler shifts and/or estimation errors whence are assumed to lie within a small fraction of the subcarrier spacing, i.e., $\left|\varepsilon_{i p}\right| \approx 0$, and $\mathbf{F}\left(\varepsilon_{i p}\right)$ in (7) can be replaced with $\mathbf{I}_{N_{c}}$, where $\mathbf{I}_{N_{c}}$ is an identity matrix of order $N_{c}$. When $\left|\varepsilon_{i p}\right| \ll 1, \mathbf{F}\left(\varepsilon_{i p}\right)$ can be viewed as a tri-diagonal matrix. In this case, $\mathbf{F}\left(\varepsilon_{i p}\right) \mathbf{v}_{i p} H_{i p}^{\iota}(0) \approx \mathbf{v}_{i p} \tilde{H}_{i p}^{\iota}(0)$ with the exceptions of the highest and lowest indexed terms. By combing these small deviation terms with $\mathbf{n}_{p}^{\iota}$ to form $\tilde{\mathbf{n}}_{p}^{\iota}$, we rewrite (7) as

$$
\mathbf{Y}_{p}^{\iota}=\sum_{i=0}^{M-1} \mathbf{v}_{i p} \tilde{H}_{i p}^{\iota}+\tilde{\mathbf{n}}_{p}^{\iota}, \iota=0,1, \cdots, N_{b}-1
$$

which can be expressed in a more compact form

$$
\mathbf{Y}_{\mathbf{p}}=\left(\mathbf{Y}_{\mathbf{p}}^{\mathbf{0}} \mathbf{Y}_{\mathrm{p}}^{1} \cdots \mathbf{Y}_{\mathbf{p}}^{\mathbf{N}_{\mathrm{b}}-\mathbf{1}}\right)=\mathbf{V}_{\mathbf{p}} \tilde{\mathbf{H}}_{\mathbf{p}}+\tilde{\mathbf{N}}_{\mathbf{p}}
$$

where $\mathbf{Y}_{\mathbf{p}}$ is the matrix obtained by stacking up the received samples from subbands within the $p$ th group, $\mathbf{V}_{\mathbf{p}}=\left(\mathbf{v}_{0 p} \mathbf{v}_{1 p}\right.$ $\left.\cdots \mathbf{v}_{(M-1) p}\right), \tilde{\mathbf{N}}_{\mathbf{p}}=\left(\tilde{\mathbf{n}}_{p}^{0} \tilde{\mathbf{n}}_{p}^{1} \cdots, \tilde{\mathbf{n}}_{p}^{N_{b}-1}\right)$, and

$$
\tilde{\mathbf{H}}_{\mathbf{p}}=\left(\begin{array}{cccc}
\tilde{H}_{0 p}^{0} & \tilde{H}_{0 p}^{1} & \cdots & \tilde{H}_{0 p}^{N_{b}-1} \\
\vdots & \vdots & \ddots & \vdots \\
\tilde{H}_{(M-1) p}^{0} & \tilde{H}_{(M-1) p}^{1} & \cdots & \tilde{H}_{(M-1) p}^{N_{b}-1}
\end{array}\right) \text {. }
$$




\subsection{Multi-user Ranging Signal Detection and Timing Offset Estimation}

The fractional frequency offset results in inter-carrier interference (ICI) to subbands in the nearby group. Let $E_{p}$ be the energy (i.e., the magnitude square of the DFT output) of the $p$ th subcarrier group excluding the highest and lowest indexed subcarriers of each subband. In the absence of signal, $E_{p}$ is a chi-square distributed with $2 N_{b}\left(N_{c}-2\right)$ degrees of freedom. When the noise variance is known, the desired false alarm probability can be achieved by selecting a proper $\eta_{0}$. We check $E_{p}>\eta_{0}$ first to see if subsequent operations are needed.

The covariance matrix of $\mathbf{Y}_{\mathbf{p}}$ is defined by $\boldsymbol{\Phi}_{\mathbf{p}}=$ $\frac{1}{N_{b}} \mathbf{Y}_{\mathbf{p}} \mathbf{Y}_{\mathbf{p}}{ }^{\mathbf{H}}$. When there are $\kappa_{p}$ RSSs in the $p$ th group, $\kappa_{p} \leq M$, we have $N_{c}-\kappa_{p}$ basis vectors which span the null space $\mathbf{U}_{\mathbf{w} \mathbf{p}}$ and can be obtained by performing singular value decomposition (SVD) on $\boldsymbol{\Phi}_{\mathbf{p}}$. Assuming $\hat{\kappa}_{p}=M$ we compute

$$
\Upsilon(d)=\frac{\left\|\alpha^{\mathbf{H}}(d) \alpha(d)\right\|}{\left\|\alpha^{\mathbf{H}}(d) \mathbf{U}_{w p} \mathbf{U}_{w p}^{\mathbf{H}} \alpha(d)\right\|}, \quad d=0, \cdots, N-1 .
$$

where $\alpha(d)=\left(1, e^{-j 2 \pi d / N}, \cdots, e^{-j 2 \pi d\left(N_{c}-1\right) / N}\right)^{\mathbf{T}}$, and find the local maximums. These peak values correspond to the ratio of the total energy to the energy projecting onto the null space. There is an one-to-one correspondence between the local peaks and the active RSS's delays. Therefore, we make a decision about $R_{i p}$ according to whether there is a peak located within $\{N i / M, N(i+1) / M\}$.

The vector $\hat{\mathbf{d}}_{\mathbf{p}}=\left(\hat{d}_{0 p}, \hat{d}_{1 p}, \cdots, \hat{d}_{(M-1) p}\right)^{T}$, which includes information about the number of the active RSSs in the $p$ th group and their delays, is obtained by the following algorithm.

1. Compute $E_{p}$ based on the received frequency domain samples. If $E_{p}<\eta_{0}$, "no signal" is declared and the algorithm terminates.

2. Arrange the frequency domain samples in matrix form $\mathbf{Y}_{\mathbf{p}}$ and set $\hat{\kappa}_{p}=M$.

3. Apply SVD to $\Phi_{\mathbf{p}}$, find the $N_{c} \times\left(N_{c}-\hat{\kappa}_{p}\right)$ matrix $\hat{\mathbf{U}}_{\mathbf{w p}}$ and then $\Upsilon(d)$.

4. Find the largest $\hat{\kappa}_{p}$ peaks of $\Upsilon(d)$ and compare these local peak values with the threshold, $\eta_{1}\left(N_{c}, N_{b}, \hat{\kappa}_{p}\right)$, which is determined by numerical optimization. If there exist $l$ peaks below the threshold then $\hat{\kappa}_{p} \leftarrow \hat{\kappa}_{p}-l$ and go back to Step 3 .

5. Based on the local peak positions, we determine if an RSS $R_{i p}$ is active and its timing offset $\hat{d}_{i p}$ is obtained by subtracting $N i / M$ from the peak position.

\section{SIMULATION RESULT AND DISCUSSION}

\subsection{Simulation Setup}

The OFDMA system parameters used in the simulations reported in this section are selected from [1] and [8]. The uplink bandwidth is $10 \mathrm{MHz}$, and the subcarrier spacing is $10.9375 \mathrm{KHz}$. The FFT size, $N$, is 1024 . ITU Vehicular A channel model is used and the sampling interval, $T_{s}$, is $89.285 \mathrm{~ns}$. We consider a cell size of radius $5 \mathrm{~km}$ so that the round-trip delay $d_{\max }=33.34 \mu s=373$ samples. $N_{g}$ is assumed to be 512 samples to satisfy the condition $N_{g}>$ $d_{\max }+L$ and each group supports $M=2$ RSSs. The subcarriers numbers and OFDM symbols used by one group for our proposal $\left(N_{c}=6, N_{b}=6, N_{g p}=4\right.$ and $\left.D_{b}=140\right)$ and two earlier schemes are summarized in Table 1 where FLM refers to the scheme proposed by Fu, $\mathrm{Li}$ and Minn [4] while SMP is an abbreviation for the method proposed by Sanguinetti, Morelli, and Poor [6].

Table 1. System Parameter

\begin{tabular}{|c|c|c|c|}
\hline & Proposed & FLM & SMP \\
\hline $\begin{array}{c}\text { Supported RSS number } \\
\text { per group, } M\end{array}$ & 2 & 2 & 2 \\
\hline $\begin{array}{c}\text { Subcarriers } \\
\text { per OFDM symbol } \\
\left(N_{c} \times N_{b}\right)\end{array}$ & 36 & 36 & 32 \\
\hline $\begin{array}{c}\text { Ranging signal duration } \\
\text { (in OFDM symbols) }\end{array}$ & 1 & 2 & 3 \\
\hline
\end{tabular}

The supported maximum speed of RSS is $120 \mathrm{Km} / \mathrm{hr}$ and carrier frequency is $2.5 \mathrm{GHz}$. The normalized residual CFOs of RSSs thus lie within the range $[-0.05,0.05]$ and are assumed to be i.i.d. for different RSSs. Because our proposed algorithm utilizes the FFT ouput data, the interference mainly come from RSSs in same group and RSSs in the nearby groups. We assume there are $K$ active RSSs in the group and $I$ RSSs in the nearby groups. Some or all of the following RSS distributions are considered: (i) $K=1, I=0$, (ii) $K=1, I=1$, (iii) $K=1, I=2$, (iv) $K=2, I=0$, and (v) $K=2, I=2$, within one ranging time-slot.

\subsection{Multi-User Detection Performance}

SNR is defined as the ratio of signal variance to noise variance in time domain. Comparisons are made with FLM and SMP ranging schemes. The results of Fig. 1 indicates that the proposed scheme performs remarkably better than FLM and SMP because of its intrinsic robustness against CFOs. In FLM scheme, the $i$ th ranging code is declared active provided that the quantity exceeds a suitable threshold $\eta$ which is proportional to the estimated noise power $\sigma^{2}$. The fractional RSSs' CFOs destroy the orthogonality in the same group. When a RSS power becomes larger, so are the interference and the false alarm probability. Hence, the performance is dominated by the interference from the same group.

In the SMP scheme, the interference from the same group is mitigated by employing MUSIC algorithm with extending one more OFDM symbol. However, the interference from nearby groups degrades the performance when the subbands assigned for different groups are adjacent. For alleviating this effect, the guard bands are required. On the other hand, our scheme can find that the RSSs in the different group do not 


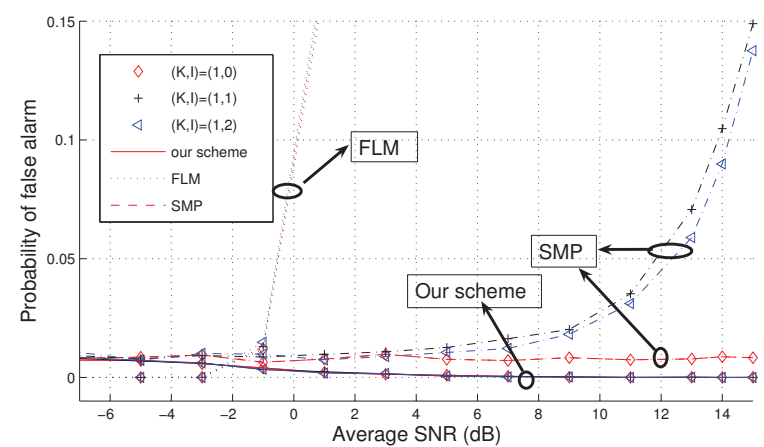

Fig. 1. Probability of false alarm as a function of average SNR

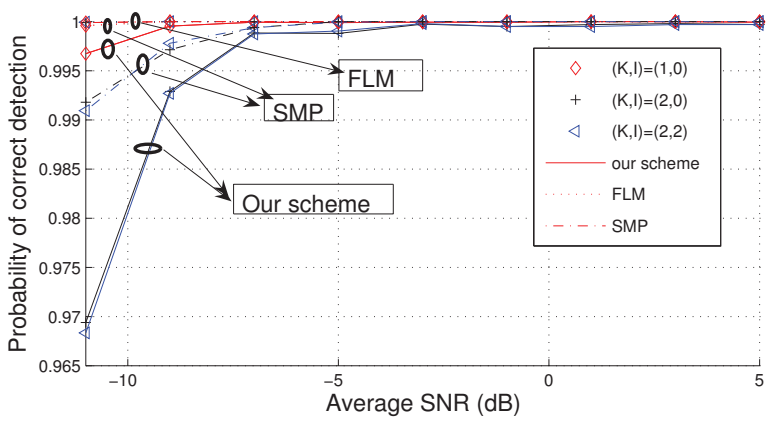

Fig. 2. Detection probability performance as a function of average SNR.

cause increase of false alarm probability even if the residual frequency offset exists. When SNR is lower, both signal and null spaces are influenced by relative large noise. When the $\mathrm{SNR}>5 \mathrm{~dB}$, the noise effect becomes insignificant. However, even for SNR $<-6 \mathrm{~dB}$, the false alarm probability is still less than 0.02 . Fig. 2 shows the probability of correct detection $\left(P_{D}\right)$ performance versus average SNR. As our ranging signal needs only one OFDM symbol the required transmission energy is less than the existing approaches that employ two or more symbols. Our proposal gives near perfect $P_{D}(\approx 1)$ even if SNR is as small as $-10 \mathrm{~dB}$. The performance degrades slightly if there are two RSSs in the same group. The reason is that the increased active RSS number reduces the dimensionality of the noise subspace. When SNR is larger than $-7 \mathrm{~dB}$, the detection performance loss of is less than 0.005 .

\subsection{Performance of Timing Estimator}

Fig. 3 plots the the timing estimation jitter, i.e., the root mean squared timing offset estimation error, as a function of the average SNR for the various RSS distributions. In each simulation run, the true transmission delays are taken randomly from the interval $\left[0, d_{\max }\right]$. In simulating the FLM scheme, we employ the SEGA algorithm with two iterations. It is observed that one RSS in one group yields better performance than two RSSs in one group. But the performance difference is just one sampling interval. We can see that for SNR larger than $1 \mathrm{~dB}$ the proposed and the FLM schemes give almost identical performance. After each RSS has suc-

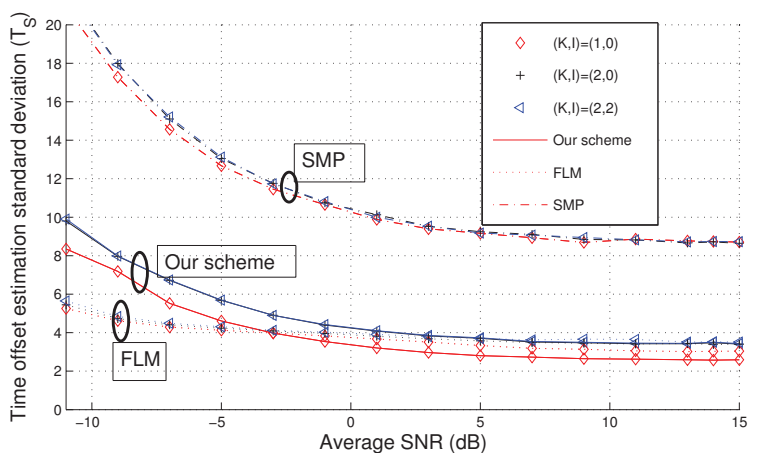

Fig. 3. Timing jitter behavior as a function of average SNR. cessfully finished initial ranging, adjusted its timing offset, the BS will be able to support more RSSs in one group for subsequent periodic ranging since most of the round-trip delay uncertainty has been removed and the $\mathrm{CP}$ length can be reduced accordingly; see (5).

\section{CONCLUSION}

A single OFDMA symbol based ranging signal design for initial ranging in a wireless mobile OFDMA system is proposed. A ranging scheme that provides accurate multiple RSS' timing estimate is presented as well. Our approach is based on the idea of projecting the received multiple ranging signals onto the null (noise) space. No information about the active RSSs' strengths are needed in our proposal. Numerical results have demonstrated that the proposed solution is capable of offering excellent detection and false alarm probabilities performance and provides a good timing estimate for multiple active RSSs.

\section{REFERENCES}

[1] IEEE LAN/MAN Standard Committee, "Air interface for fixed and mobile broadband wireless access systems," IEEE 802.16e-2005.

[2] J. Krinock, et al. "Comments on OFDMA ranging scheme described in IEEE 802.16ab01/01r1," document IEEE 802.abs-01/24.

[3] X. Zhuang, et al., "Ranging improvement for $802.16 \mathrm{e}$ OFDMA PHY," document IEEE 802.16e-04/143r1.

[4] X. Fu, et al. "A new ranging method for OFDMA systems," IEEE Trans. Wireless Commun, vol. 6, no. 2, pp. 659-669, Feb. 2007.

[5] K. C. Lin, et al., "Ranging Code Design for IEEE 802.16m,” IEEE C802.16m-08_329, May 5, 2008.

[6] L. Sanguinetti, et al., "An improved scheme for initial ranging in OFDMA-based networks," in Proc., ICC2008, Beijing, China, pp. 3469-3474, May 2008.

[7] M. Morelli, et al. "Synchronization techniques for orthogonal frequency division multiple access (OFDMA): A tutorial review," Pro. IEEE, vol. 95, no. 7, pp. 13941427, Jul. 2007.

[8] IEEE 802.16 Broadband Wireless Access Working Group, "Draft IEEE 802.16m evaluation methodology," IEEE 802.16m-07/037r1. 